\title{
The Application of Magnetic Sensors in Self-Contained Local Positioning
}

\author{
Chengliang Huang and Xiao-Ping Zhang \\ Ryerson University \\ Canada
}

\section{Introduction}

Nowadays, GPS (Global Positioning System) has been widely used to position airplanes, ships, automobiles, migrating animals, and human beings, etc. However, GPS has its own limitations. Other than the user groups for whom GPS is operated, there is no service level agreement with anyone else. As more and more people become dependant on GPS in more and more countries, the political control of GPS raised doubt about its value. Moreover, in the event of system failure, equipment depending on GPS, such as airplanes or ocean-going vessels, needs alternative positioning systems. Furthermore, GPS relies on complicated infrastructure and cannot provide sufficient accuracy, stability and coverage for environments such as indoors and highly urbanized areas (known as "urban canyon") due to the lack of lineof-sight (LOS) to the satellites of GPS. As a result, most commercial and social activities taking place in such environment can not benefit from positioning services offered by GPS.

Local positioning systems (LPS) are intended to provide a back-up for applications where GPS does not perform sufficiently to the requirements of the users. As indicated by the name, LPS can only provide positioning services within a limited area. Unlike GPS, LPS provides positioning services in communities, campuses, urbanized areas and inside buildings. LPS can be categorized into two groups: indoor positioning systems and outdoor positioning systems. The former provides positioning services inside buildings while the latter provides the services outdoors.

LPS can provide positioning services in a wide range of applications, including personal guidance, people-finding, mobile information, moving object management and so on. LPS is also essential for augmented reality and ubiquitous computation. Typical applications of LPS are as follows personal navigation assistances (PNA), personal guidance (PG), LPS tags, people-rescue and intelligent transportation services (ITS).

PNA is a portable electronic device with both positioning function and navigation capability. A PNA based system is more flexible than GPS in providing optimal route descriptions for pedestrians because they only rely on the same maps for both in-vehicle and on-foot scenarios. On the other hand, as an interactive portable device, PG can help people with effective routing, or real-time reservation, and journey updating to inform the concerned parties of the latest situation on the journey. With this service, traveling will be easier and safer for people. 
LPS can find the location of people equipped with an LPS tag, such as business men traveling in urban areas, workers working on a construction site, fire fighters in operation, such that the pertinent activities can actively monitored and coordinated for better effectiveness, efficiency and safety. An important application of LPS in public services is people-rescue, such as urban search and rescue (USAR). When disasters such as earthquakes, cyclones, tornadoes and floods occur, some specialized organizations, governments and private companies will dispatch task forces immediately. The task force will firstly locate and extricate victims entrapped, then provide and conduct first aid care. Those victims may be entrapped in confined spaces such as collapsed structures and trenches, mines and transportation accidents. Obviously, the positioning of the victims is critical for an efficient rescue action.

A typical application of LPS in transportation is so-called ITS. This new technology is still being developed to control traffic jam and improve road safety. ITS also reduces vehicle wear, travel time, and fuel consumption through managing competing factors such as vehicle type, load, and routes. Other than conventional services such as electronic toll collection and emergency vehicle notification, sophisticated services are also provided to control urban traffic and to guide drivers with automatic routes for optimal performance. LPS, integrated with GPS, makes these services possible.

In the long run, both the technology and society environments will be changed greatly. Integrated circuits (IC) will be faster, smaller and cheaper according to Moore's law. The positioning will be more accurate and robust with advanced algorithms and new sensors. Building automation and indoor three-dimensional (3D) dynamic mapping will illustrate and control the whole building environment in much greater details such that both the quality of indoor environment and the operation cost-effectiveness will be significantly improved. Virtual world and real world will be merged to create new sports, games, entertainment and arts. People will have more wealth, enjoying more social networking, and receive more cares. As a result, LPS will surely have more and more applications. Personal positioning devices will be popular to every working persons and school students. Value added services will be provided through LBS (Location Based Services) and ITS. GIS (geographic information system) and LPS will be combined to provide quick emergency response and automatic rescuing. Much smarter supply chain management, traffic management, and work force management will be available to businesses. LPS may induce a commercial exploitation in the future.

\section{Strap-down inertial positioning}

Some LPS systems reply on certain infrastructures and are difficult to setup and maintain. They are not suitable for applications that require quick setup, such as fire fighting operations, and emergency rescue services. We recommend self-contained LPS to locate objects residing or moving within a covered area. These objects are equipped with intelligent sensor modules, normally in form of microelectromechanical system (MEMS) component that can sense both the direction and speed of movement and compute the position of the objects within a given coordinate system. In this section we introduce a selfcontained pedestrian tracking technique for the establishment of a complete infrastructurefree positioning system. The technique is the synergism of two existing technologies of dead 
reckoning and inertial positioning. In the implementation of such a technology, the magnetic sensors take an important role in achieving acceptable performance.

\subsection{Dead reckoning}

Examples of existing self-contained LPS systems include dead reckoning systems for marine navigation (McBrewster, 2009), wheel sensing systems for automobiles (Thiessen \& Dales, 1983), and pedometers for human beings (Chiang, 2007). Dead reckoning is a method of estimating an object's present position by projecting its courses steered and speeds over ground from the last known position. The dead reckoning position is only a rough approximation because it does not consider the effect of leeway, currents, helmsman error, or gyro error.

All dead reckoning systems compute the position of an object based on the last known position and the relative movement from that position. The relative movement is calculated according to the direction and speed of motion. This principle is employed in the aforementioned three existing self-contained LPS. Despite the advantage of independence on complicated infrastructure, these systems normally suffer from low accuracy.

\subsection{Inertial positioning}

An inertial positioning system (IPS) indirectly obtains the speed and direction, or displacement, through integrating the measured accelerations and the angular velocity of an object over time. Accelerometers and gyroscopes are used to continuously measure and record translational and rotational motions. Thus inertial positioning and dead reckoning are different in the data acquired (acceleration v. s. speed, angular velocity v. s. course direction) and the devices used to measure these data (e. g. accelerometer v. s. speedometer). For a dead reckoning system, the distance can also be acquired through direct estimation. For example, in a pedometer, the step length is simply estimated, not measured or calculated. The distance is estimated by multiplying the estimated step length and the number of steps counted. Generally speaking, inertial positioning, or inertial navigation, is a modern technology superseding the dead reckoning, a relatively older technology.

A traditional inertial positioning system is gimbaled (Sarton and George, 1959). A mechanical device called gimbal-stabilized platform is used to establish a reference system in vehicles such as submarines, surface vehicles, aircrafts and space crafts. The sensors, the gyroscopes and accelerometers, or pick-ups, are mounted up on the stabilized platform to sense specific forces. The advantage of gimbaled systems is that the computation of position, velocity, orientation and angular velocity is less complex.

As shown in Fig. 1, with the initial local velocity $\mathbf{v}^{1}(0)$ and initial local displacement $\mathbf{s}^{1}(0)$ of an object known respectively, we are able to track the position of the object by subtracting

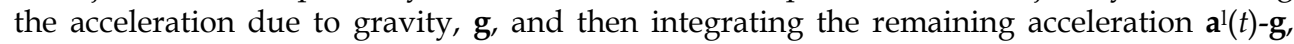
once over a time period $t$ to obtain velocity, and twice to obtain displacement in the local geographic frame (l-frame):

$$
\begin{gathered}
\mathbf{v}^{1}(t)=\mathbf{v}^{1}(0)+\int_{0}^{t}\left(\mathbf{s}^{1}(\tau)-\mathbf{g}\right) \mathrm{d} \tau \\
\mathbf{s}^{1}(t)=\mathbf{s}^{1}(0)+\int_{0}^{t} v^{1}(\tau) \mathrm{d} \tau
\end{gathered}
$$




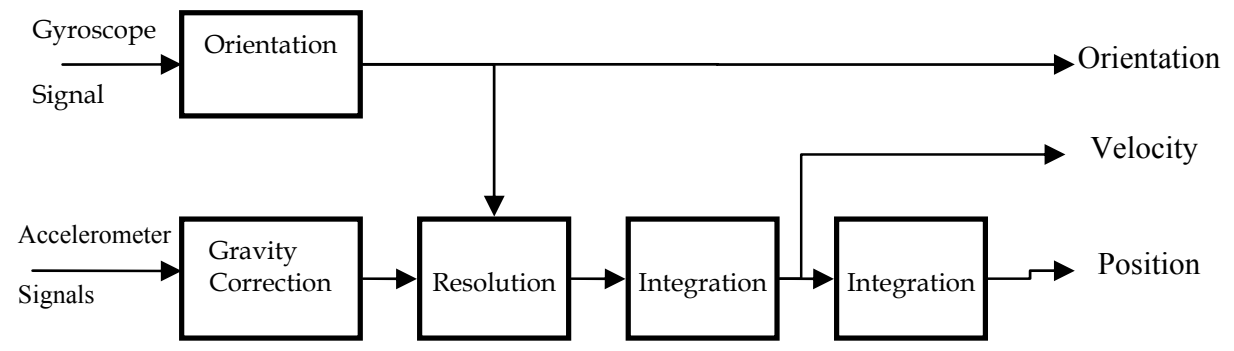

Fig. 1. Traditional inertial positioning algorithm

Using the rectangular rule, the above integration can be implemented in discrete-time form , denoting $\delta t$ as the integration interval:

$$
\begin{gathered}
\mathbf{v}^{1}(t+\delta t)=\mathbf{v}^{1}(t)+\left(\mathbf{a}^{1}(t+\delta t)-\mathbf{g}^{1}\right) \cdot \delta t \\
\mathbf{s}^{1}(t+\delta t)=\mathbf{s}^{1}(t)+\mathbf{v}^{1}(t+\delta t) \cdot \delta t
\end{gathered}
$$

Unfortunately, there are several problems with the gimbaled systems. Other than frictions from bearings and the dead zones of motors, extra power is needed to align the platform with the navigational frame. Moreover, gimbaled systems need high quality electromechanical parts including motors, slip rings and bearing, recalibration which is difficult, and regular maintenance by certified personnel in a clean room through a lengthy recertification process. Consequently, the traditional position systems are mostly used for airplanes, vessels, and intercontinental ballistic missiles.

\subsection{Strap-down inertial positioning}

The strap-down IPS replaces the traditional gimbaled system for low-cost applications. The accelerometers and rate gyroscopes are rigidly mounted in the body of a tagged object thus there is no relative movement between them. This is a major hardware simplification compared with the stabilized platform in tradition system. The most significant advantage of the strap-down IPS in comparison with the gimbaled IPS is the considerably reduced size and weight, which normally result in lower cost, power consumption, and hardware complexity.

However, the processing of inertial navigation is more sophisticated. Fundamentally, for IPS, a number of Cartesian coordinate reference frames, such as the body frame (b-frame), positioning frame and local geographic frame (l-frame) have to be rigorously defined and precisely related. We define the b-frame as an orthogonal axis set, which is aligned with the roll, pitch and yaw axes of the body to be positioned. We also define the positioning frame as an l-frame which has its origin at the original position for positioning and axes aligned with the directions of north, east and the local vertical (down). In a strap-down IPS, the computational complexity is increased because output data are measured in the b-frame rather than the l-frame. An algorithm has to be applied to keep track of the orientation of the sensor module (and the object) and rotate the measurements from the b-frame to the l-frame. Fig. 2 shows this procedure. 


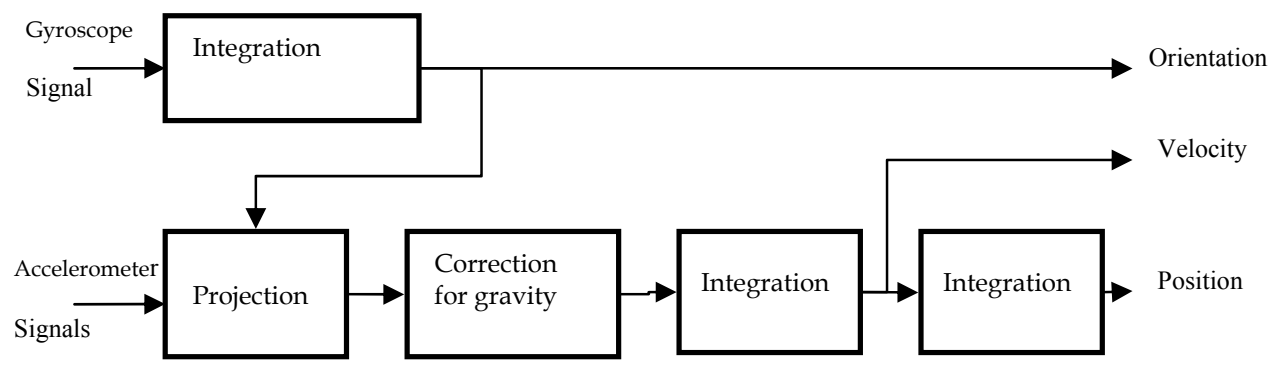

Fig. 2. Strap-down inertial navigation algorithm

\subsubsection{Reference frames and rotations}

Denoting axes $x^{1}, \mathbf{y}^{1}$, and $z^{1}$ respectively represent north, east and down in the l-frame fixed to the surface of the earth, the orientation of the device will be estimated in this coordinate frame. Axes $x^{\mathrm{b}}, \mathbf{y}^{\mathrm{b}}$ and $z^{\mathrm{b}}$ represent the orthogonal axes in the b-frame.

In order to describe the components of a vector of arbitrary orientation in l-frame with respect to the original b-frame, or, in a new frame with respect to an old frame, after a certain rotation, a few rotation representations must be applied. We use the direct cosines representation to develop algorithms for tracking the orientations of sensors. To do this, a direct cosine matrix (DCM) has to be established by transformation of three sequential rotations from the original axes in the l-frame to the new body axes. These rotations continue as a $\Psi$ rotation about $z$; a $\theta$ rotation about $y$, resulting from the first rotation; and, finally, a $\phi$ rotation about $x$, resulting from the second rotation. The resulting DCM is as follows:

$$
\mathbf{R}=\left(\begin{array}{ccc}
\cos \theta \cos \psi & -\cos \phi \sin \psi+\sin \phi \sin \theta \cos \psi & \sin \phi \sin \psi+\cos \phi \sin \theta \cos \psi \\
\cos \theta \sin \psi & \cos \phi \cos \psi+\sin \phi \sin \theta \sin \psi & -\sin \phi \cos \psi+\cos \phi \sin \theta \sin \psi \\
-\sin \theta & \sin \phi \cos \theta & \cos \phi \cos \theta
\end{array}\right)
$$

\subsubsection{Tracking the orientation of a moving object}

The orientation of a moving object is tracked by integrating the angular velocity signal obtained from rate gyroscope in the swing phase of walking.

At a time instant $t$, let $\omega=\left[\begin{array}{lll}\omega_{\mathrm{x}} & \omega_{\mathrm{y}} & \omega_{\mathrm{z}}\end{array}\right]^{\mathrm{T}}$ be the corresponding angular velocity sample, denote the norm of angular velocity as $\xi$,i.e., $\xi=\left\|\omega_{\mathrm{x}}, \omega_{\mathrm{y}}, \omega_{\mathrm{z}}\right\|$, and a matrix

$$
\boldsymbol{\Omega}=\left(\begin{array}{ccc}
0 & -\omega z & \omega y \\
\omega z & 0 & -\omega x \\
-\omega_{y} & \omega x & 0
\end{array}\right)
$$

With a short sampling interval $\delta t$, the DCM at time $t, \mathbf{R}(t)$, can be calculated from the DCM at time $t-\delta t, \mathbf{R}(t-\delta t)$, according to below equation

$$
\mathbf{R}(t)=\mathbf{R}(t-\delta t) \cdot\left(\mathbf{I}+\frac{\sin (\xi \delta t)}{\xi} \boldsymbol{\Omega}+\frac{1-\cos (\xi \delta t)}{\xi^{2}} \boldsymbol{\Omega}^{2}\right)
$$


With the DCM updated, it becomes possible to project the acceleration signal $\mathbf{a}^{\mathrm{b}}(t)$ from the accelerometers in the b-frame into acceleration $\mathbf{a}^{\mathrm{l}}(t)$ in the l-frame:

$$
\mathbf{a}^{1}(t)=\mathbf{R}(t) \cdot \mathbf{a}^{\mathrm{b}}(t)
$$

After the local acceleration is obtained, the velocity and the displacement of the object can be determined according to (1) and (2) respectively to locate the object.

\section{Inertial sensors}

As mentioned above, inertial positioning relies on the measurement of accelerations and angular velocities. The estimate of changes in rotated angles can be obtained through integrating the angular velocity from a gyroscope over time. The estimate of changes in velocities and positions in the l-frame can be obtained through integrating the local acceleration over time once and twice respectively. The local acceleration is the projection of body acceleration, measured from accelerometers, onto the l-frame.

\subsection{Gyroscopes and accelerometers}

A wide range of sensors can detect or measure angular movement. These devices range from the conventional mechanical gyroscopes and optical gyroscopes, to the ones based on atomic spin.

An accelerometer measures the physical acceleration it experiences relative to freefall, not to the coordinate systems. There are many types of accelerometers. Generally, all these devices are amenable for strap-down applications. However, the accuracy ranges widely from micro-g to fractions of $g$ due to the variety of designs. Acceleration can be internally measured without using any external references, which are needed for the measurement of velocity. This is why it is preferable to develop algorithms based on acceleration for LPS.

MEMS technology, used to form structures with dimensions in the micrometer scale, is now being employed in manufacturing state-of-the-art MEMS-based inertial sensors.

\subsection{Performance evaluation}

Practically, the measuring errors of accelerometer and rate gyroscope result in errors during positioning. Assuming the average output from an accelerometer when it is not undergoing any movement is $b_{\mathrm{a}}$, the accumulated positioning error after double integration over time period $t$ is $e_{\mathrm{s}}(t)=b_{\mathrm{a}} t^{2} / 2$. This means that the positioning error caused by a constant bias of accelerometer increases quadratically through double integration over time. Such an error is called integral drift.

Similarly, assuming the offset of the output angular velocity from true value is $b_{\mathrm{g}}$, the error of a gyroscope over time period $t$, we have $e_{\mathrm{g}}(t)=b_{\mathrm{g}} t$. This linearly increasing angular error, i.e. orientation error, can cause the error of the rotation matrix $\mathbf{R}(t)$. The resulting incorrect projection of acceleration signals onto the global axes causes two problems. Firstly, the acceleration is integrated in the incorrect direction, causing interaction of velocity and position between (not along) axes. Secondly, acceleration due to gravity can not be removed 
completely. The residue acceleration originated from gravity will become a "bias" to the true acceleration due to movement of the object.

In the strap-down navigation algorithm, the acceleration due to gravity, $\mathbf{g}$, is deducted from the globally vertical acceleration signal before integration. When there are angular errors, there are tilt errors. Here a tilt error refers to the angle between the estimated vertical direction and true vertical direction. The tilt error $e_{\phi}$ in radian will cause the projection of gravity onto the horizontal axes, resulting in a component of the acceleration due to gravity with magnitude: $e_{\mathrm{a}}^{\mathrm{h}}=\mathrm{g} \times \sin e_{\phi}$. This component can be treated as a residual bias due to gravity, remaining in the globally horizontal acceleration signals. In the mean time, in globally vertical axis, there is a

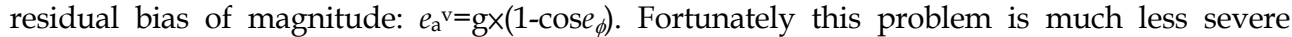
because for small $e_{\phi}$, we have $e_{\mathrm{a}} \mathrm{v} \approx 0$. Therefore, a small tilt error will mainly cause positioning error in the globally horizontal plane.

In some cases, Such as human walk, the mean absolute acceleration measured is much smaller than the magnitude of gravity. As a contrast, a tilt error of $0.05^{\circ}$ can cause a component of the acceleration due to gravity with magnitude near $\mathrm{g} / 1000$. This residue bias can cause a positioning error of 15.4 meters after only a minute of integration, or error of 0.49 meter after only 10 seconds (as demonstrated by the red dashed line in Fig. 3). Therefore, gyroscope errors, which propagate in the positioning algorithm, are critical errors affecting the accuracy of pedestrian tracking. Before the development of the algorithm presented in this chapter, it was believed that positioning with data from inertial sensors was not possible due to the quadratic growth of errors caused by sensor drift during double integration.

\section{Integration of PDR with inertial positioning}

Precise tracking of people, especially of first responders, in a harsh environment, remains an open research area. For a practical person tracking system, the measuring device of the system should be portable or wearable for the person being tracked. Inertial positioning can be applied in people tracking theoretically. However, the integral drift of accelerometers and gyroscopes, as well as the tilt errors of gyroscopes, make the inertial positioning not practical. As a result, as mentioned earlier, the characteristic of the movement of the person should be incorporated to improve positioning accuracy. If pedestrian dead reckoning (PDR) is integrated with IPS, the performance will be significantly improved, as shown is Fig. 3.

\subsection{Pedestrian dead reckoning}

PDR is the application of dead reckoning in pedestrian tracking. Due to the difficulty in measuring the speed of a walking person directly, PDR estimate a pedestrian's present position based on estimated step length and heading from the last known position. Using probability models, Mezentsev et al (2005) confirm that the main source of errors in a PDR system is related to the estimation of the step length and the heading. Different random noise models can be applied to heading estimation and step length estimation (Mezentsev et al, 2005). Suppose the modeled mean step length $s$ is a constant, and the $i$ th length error $\omega_{i}$ is normally distributed with a standard deviation $\sigma$, then the true $i$ th step length satisfy $s_{i}=s+\omega_{i}$. Assuming the step errors are uncorrelated during a walk, the distance error variance after $N$ steps is 


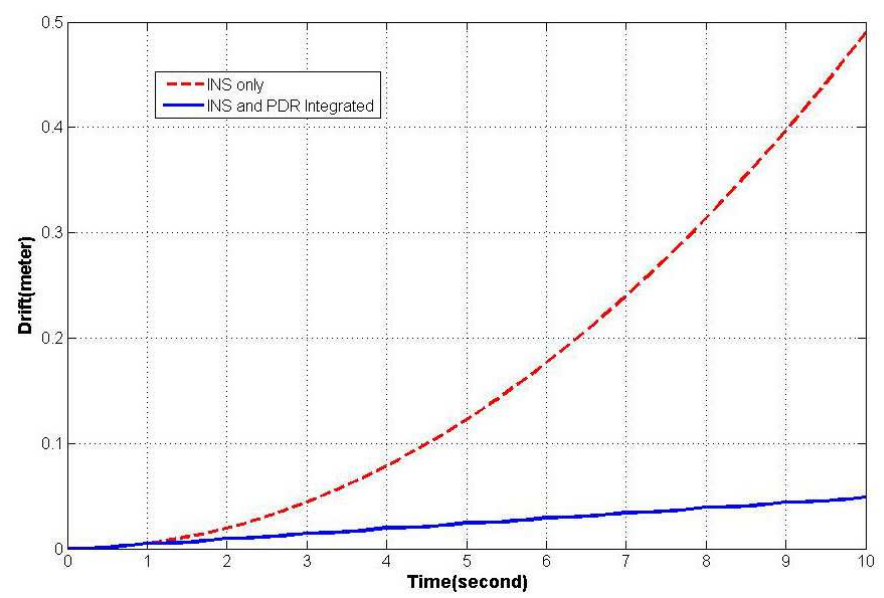

Fig. 3. Performance of positioning with IPS and PDR integrated

$$
\sigma_{s}^{2}=\operatorname{Var}\left(\sum_{k=1}^{n} \omega_{k}\right)=\sum_{k=1}^{n} E\left(\omega_{k}^{2}\right)=N \sigma^{2}
$$

Hence, the error variation equals the product of the step count and the variation of the driving noise. Accordingly, the position error maximizes when the pedestrian walk continuously along a straight line with a constant step length error. Therefore, if not assisted by other methods, PDR is not able to provide precise positioning. For further improvement of accuracy, the kinetics of human walk is investigated.

\subsection{Human gait and phase detection}

According to Rose and Gamble (1994), the human motion during a walk is cyclical and repeatable. Such a basic pattern is significantly constant among individuals. The gait cycle during human walk comprises two phases: a stance phase and a swing phase. In the stance phase, a foot keeps in touch with the ground from the time when the heel strikes the ground to the time when the toes leave the ground. The swing phase starts when the foot is taken away and carried forward. When the foot touches the ground again, the swing phase ends and the next stance phase starts. Each gait cycle is completed and restart in such a way. The other foot performs the same process, but lags $180^{\circ}$ behind. Fig. 4 shows how the stance phase is started and ended. For a certain point at the front part of sole, its ground speed is almost zero from $T_{3}$ to $T_{5}$ (the period $\Delta T$ ). If this time period $\Delta T$ can be dependably detected every stride, referential data may be acquired and used to reset computations and get rid of accumulating errors, the critical problem in pedestrian tracking.

Huang et al (2010) advocate the integration of inertial navigation and pedestrian dead reckoning. With the stance phase detected, the velocity and angles can be updated in the stance phase and swing phase respectively. Thus both the integral drift of velocity and positions, as well as the tilt error caused by integral errors of rotated angles, can be greatly reduced. The positioning performance of the integration is illustrated in Fig. 3. The details of these updates are explained in the sub-sections that follows. 


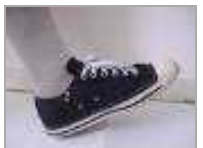

$\mathrm{T}_{1}$

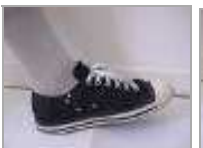

$\mathrm{T}_{2}$

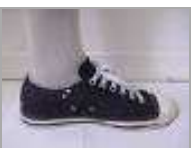

$\mathrm{T}_{3}$

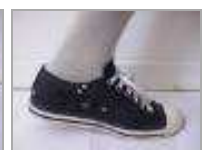

$\mathrm{T}_{4}$

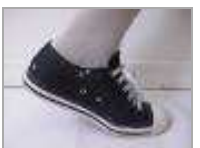

$\mathrm{T}_{5}$

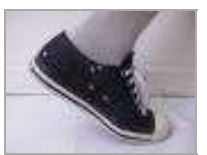

$\mathrm{T}_{6}$

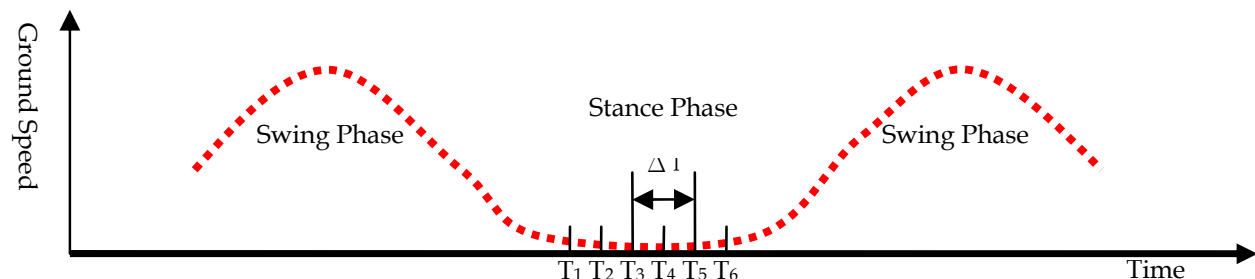

Fig. 4. Stance phase in a stride

In order to detect stance phase we can use sensor data, including all three components of angular velocity, acceleration, and even magnetic field, provided by a typical inertial/magnetic sensor module. Because the y-axis angular rate is the most significant indicator of stride events, Huang et al (2010) use the y-axis angular rate to detect the stance phases instead.

\subsection{Zero-Velocity update and swing phase integral error reduction}

Theoretically, the velocity in the l-frame is zero in the stance phase. In practice, zero-velocity updates (ZUPT) are performed when the acceleration and rate gyro measurements drop below empirically determined thresholds for a certain period of time. The main purpose of these updates is to mitigate both movement measured in the stance phase of a gait and increasing integral drifts. ZUPT is used by Stiring and Fyfy (2005), Mezentsev and Lachapelle (2005). Stirling and Fyfy stopped integrating and reset the velocity before each swing phase. The positioning accuracy of their system is of 10 to 20 percent of distance traveled.

In order to obtain a higher tracking accuracy, Huang et al (2010) further use additional methods to remove integral drifts. Some methods should be applied in the swing phase of each stride.

\subsection{Orientation update using magnetic sensors}

Huang et al (2010) update orientation using magnetic field signals from magnetic sensors, i.e., magnetometers.

In order to determine the orientation of the sensor module for rotation representation and transformation between the l-frame and the b-frame, the Euler angles must be determined. In the swing phases, the Euler angles are calculated through integration of the angular velocity over time assuming that the original orientation of the sensor module is known. However, the original orientation is usually unknown in reality. In addition, the gyroscope signal summed during integration is perturbed by gyroscope bias. Thus the accuracy of the 
orientation will decay overtime. Therefore, Huang et al (2010) use magnetometers to detect the magnetic field of the earth. Using data from magnetometers, together with gyroscopes and accelerometers, the orientation in the beginning of each human walk can be found. Similarly, the orientation of the sensor module in stance phase, when the sensor module is in a near-static status, can be updated in each gait. With the updates of orientation in stance phases, the integral drifts of rotated angles, which increase linearly over time, are greatly reduced.

To find the near-static orientation, the Euler angles $\psi, \theta$ and $\varphi$ must be calculated. Because gyroscopes cannot be used in a near-static status, signals from accelerometers and magnetometers have to be used to calculate the orientations of the sensor module. However, these angles cannot be obtained directly. To resolve this problem, the tilts and heading of the sensor module need to be investigated.

Both $\mathrm{x}$-tilt $\alpha$ and y-tilt $\beta$ of the sensor module, or the sensor on it, as shown in Fig. 5, can be found simultaneously using acceleration outputs of all three axes as below:

$$
\begin{aligned}
& \alpha=\operatorname{atan} 2\left(a_{x}, \sqrt{a_{y}^{2}+a_{z}^{2}}\right) \\
& \beta=\operatorname{atan} 2\left(a_{y}, \sqrt{a_{x}^{2}+a_{z}^{2}}\right)
\end{aligned}
$$

where the two-argument function, atan2, is a variation of the arctangent function. For any real arguments $x$ and $y$, which are not both equal to zero, atan2 $(y, x)$ is the angle in radians between the positive $x$-axis of a plane and the point given by the coordinates $(x, y)$ on it.

The heading of a pedestrian, thus that of the sensor module on him/her, can be calculated using the $x$-axis and $y$-axis magnetic field outputs if the sensor module is level in a horizontal plane. As shown in Fig. 6, only the $x$ and $y$ components of the Earth's magnetic field, are used when determining the heading of sensor module. The heading $\gamma$ is defined by:

$$
\gamma=\operatorname{atan} 2\left(h_{\mathrm{y}}, h_{\mathrm{x}}\right)
$$

where $h_{\mathrm{x}}, h_{\mathrm{y}}$ and $h_{\mathrm{z}}$ are components of magnetic field on $\mathrm{x}, \mathrm{y}, \mathrm{z}$ axis respectively.

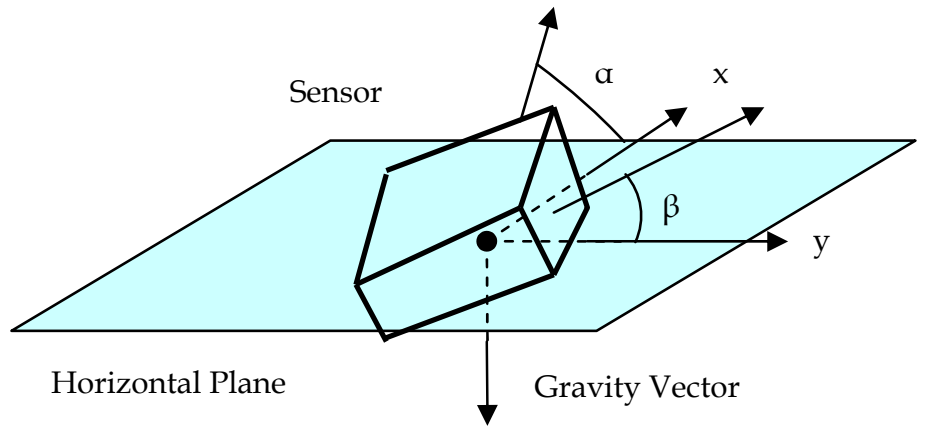

Fig. 5. x-tilt and y-tilt assignments relative to ground 


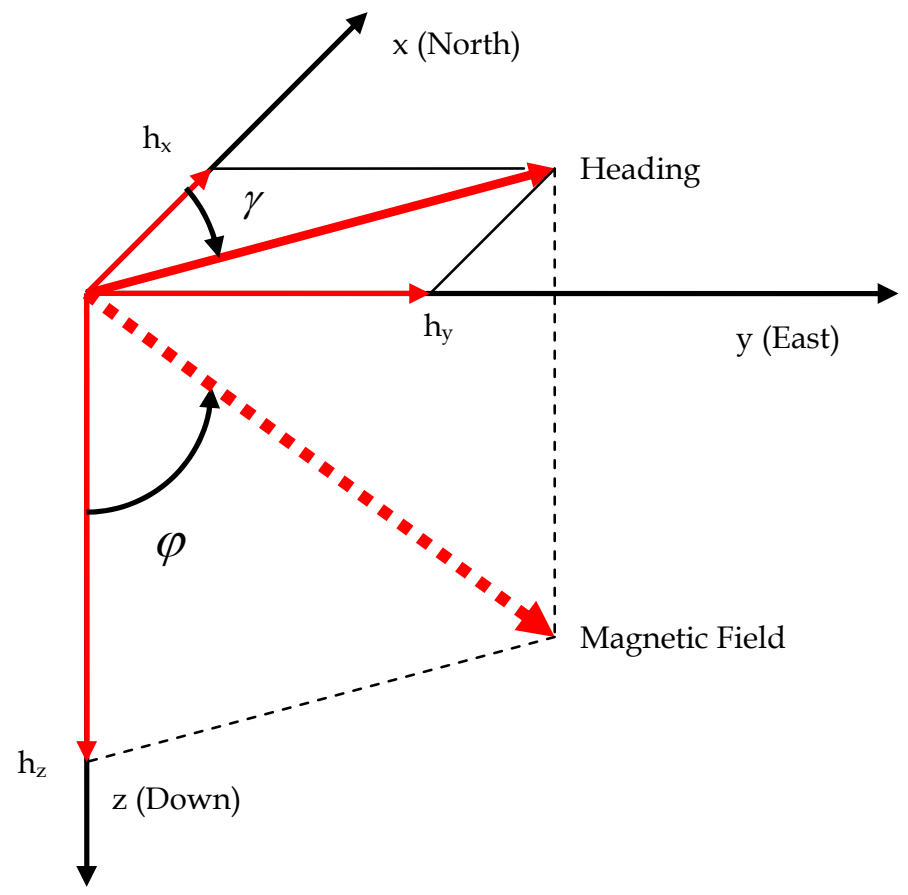

Fig. 6. Heading defined in the horizontal x-y plane

No matter whether a person is standing or walking, the sensor module attached to the human body is not confined to a flat and level plane. This makes it more difficult to determine the heading direction, because the tilt angles of the $x$-axis and the $y$-axis are always changing and the sensor module cannot stay horizontal to the earth's surface. Errors caused by tilt angles can be large if not compensated.

To compensate the tilt of the sensor module when it is in an arbitrary position, the l-frame is transformed into the $b$-frame through three rotations with Euler angles $\phi, \theta$ and $\phi$ in sequence. The DCMs of the rotations are denoted as $\mathbf{R}_{1}(\psi, z), \mathbf{R}_{2}(\theta, y)$ and $\mathbf{R}_{3}(\phi, x)$ respectively. These Euler angles are related to the tilt but they are not all the tilts of the sensor module. The algorithm (Huang et al 2010) is as follows.

Rotations of the gravity vector from the l-frame to the $b$-frame are given by

$$
\mathbf{g}^{\mathrm{b}}=\mathbf{R}^{\mathrm{T}} \cdot \mathbf{g}^{l}=\left[\begin{array}{lll}
-\sin \theta & \sin \phi \cos \theta & \cos \phi \cos \theta
\end{array}\right]^{\mathrm{T}}
$$

where $\mathbf{g}^{\mathrm{b}}=\left(g_{\mathrm{x}} \mathrm{g}_{\mathrm{y}} \mathrm{g}_{\mathrm{z}}\right]^{\mathrm{T}}$ and $\mathbf{g}^{\mathrm{l}}=\left(\begin{array}{lll}0 & 0 & 1\end{array}\right]^{\mathrm{T}}$. Therefore, we have

$$
\theta=\operatorname{atan} 2\left(g_{x}, \sqrt{g_{y}{ }^{2}+g_{z}^{2}}\right)
$$


This equation is actually the same as equation (10) if the sensor module is not moving. Using equation (13) and (14), we obtain

$$
\phi=\operatorname{atan} 2\left(g_{y} \operatorname{sign}(\cos \theta), g_{z} \operatorname{sign}(\cos \theta)\right)
$$

This equation is different from equation (11). Hence the $\phi$ angle of roll is not the y-tilt angle $\beta$. y-tilt should not be used as roll for orientation calculation and magnetometer compensation.

After both pitch $\theta$ and roll $\phi$ are found from acceleration data, the attitude, or yaw $\psi$, can be found from magnetic field data by solving the following simultaneous equation:

$$
\mathbf{m}^{\mathrm{b}}=\mathbf{R}^{\mathrm{T}} \cdot \mathbf{m}^{1}
$$

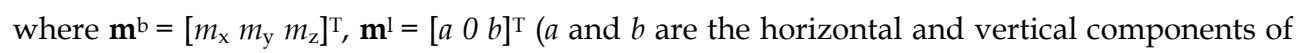
the magnetic field of the earth vector) and $\mathbf{R}^{\mathrm{T}}=\mathbf{R}_{1} \mathrm{~T}(\psi, \mathrm{z}) \mathbf{R}_{2}{ }^{\mathrm{T}}(\theta, \mathrm{y}) \mathbf{R}_{3}{ }^{\mathrm{T}}(\phi, \mathrm{x})$. Hence we get

$$
\mathbf{R}_{1}^{\mathrm{T}}(\psi, \mathrm{z}) \cdot \mathbf{m}^{1}=\mathbf{R}_{2}^{\mathrm{T}}(\theta, \mathrm{y}) \cdot \mathbf{R}_{3}^{\mathrm{T}}(\phi, \mathrm{x}) \cdot \mathbf{m}^{\mathrm{b}}
$$

As illustrated in Fig. 6, $h_{\mathrm{x}}, h_{\mathrm{y}}$ and $h_{\mathrm{z}}$ are the projections of magnetic field on the horizontal plane and the $z$-axis (pointing downward) respectively, i.e., $\mathbf{h}^{\mathrm{b}}=\left[h_{\mathrm{x}} h_{\mathrm{y}} h_{\mathrm{z}}\right]^{\mathrm{T}}=(\operatorname{acos} \psi,-\operatorname{asin} \psi]^{\mathrm{T}}$. Then the compensated magnetic field can be obtained as below:

$$
\begin{gathered}
h_{x}=m_{x} \cos \theta+m_{y} \sin \theta \sin \phi+m_{z} \sin \theta \cos \phi \\
h_{y}=m_{y} \cos \phi-m_{z} \sin \phi \\
h_{z}=m_{x} \sin \theta+m_{y} \cos \theta \sin \phi+m_{z} \cos \theta \cos \phi
\end{gathered}
$$

Accordingly, the heading on the horizontal plane, or the Euler angle yaw, can be defined as

$$
\psi=\operatorname{atan} 2\left[-h_{\mathrm{y}}, h_{\mathrm{x}}\right]
$$

The angle $\psi$ calculated in equation (21) is actually a compass reading, i.e., the heading relative to magnetic north. To get the true heading, denoted as $\psi_{\mathrm{T}}$ here, the magnetic declination $D$ must be deduced from the compass reading $\psi$ :

$$
\psi_{T}=\psi-D
$$

where $D$ is the difference between magnetic north and true north. This difference is caused by the tilt of the earth's magnetic field generator relative to the earth's spin axis.

\section{Experimental study}

To implement the methods introduced in the Section 4,. A commercial sensor module shall be carefully selected And then mounted to on a person rigidly. The result of the on-site pedestrian tracking is promising. 


\subsection{Sensor module selection}

Many factors, including size, cost, dynamic range, sampling rate and bias, have to be considered in choosing an appropriate sensor module. Firstly, the size of the sensor module should be small enough to be installed unobtrusively on footwear. Consequently, all 3D gyroscopes, 3D accelerometers and 3D magnetometers should be integrated into, actually orthogonally mounted in, this inertial/magnetic sensor module. Secondly, the price of this sensor module should be low enough to meet budget constraints for the targeted applications. Thirdly, the dynamic range of the sensor module should meet the requirements of the applications. Fourthly, the sampling rate should be high enough to avoid sampling errors. The sampling rate of the sensors should be at least $100 \mathrm{~Hz}$. Finally, because of the sensitivity of sensors to temperature, each sensor in a module should be individually compensated for bias, which is a function of temperature.

With all these considerations an inertial measurement unit (IMU) was selected to build the prototype for testing. The IMU provides serial digital outputs of 3D acceleration, 3D angular velocity, and 3D magnetic field.

\subsection{Sensor module mounting}

In the experiments, the sensor module is mounted on the arch, i.e., the upper of closed footwear, as shown in Fig 7. The installation of the sensor should ensure that the relative movement between the sensor module and the foot-ware is minimized.

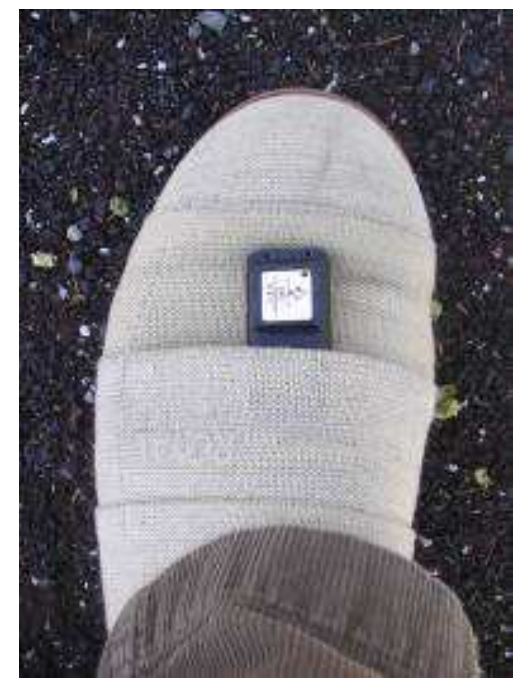

Fig. 7. Sensor Module Mounting

\subsection{Field experiment}

Using the methods introduced in Section 4 and the sensor module selected in Section 5.1, several experiments have been conducted at different paces in different places and different times. Most of these experiments are two-dimensional (2D) tests. 


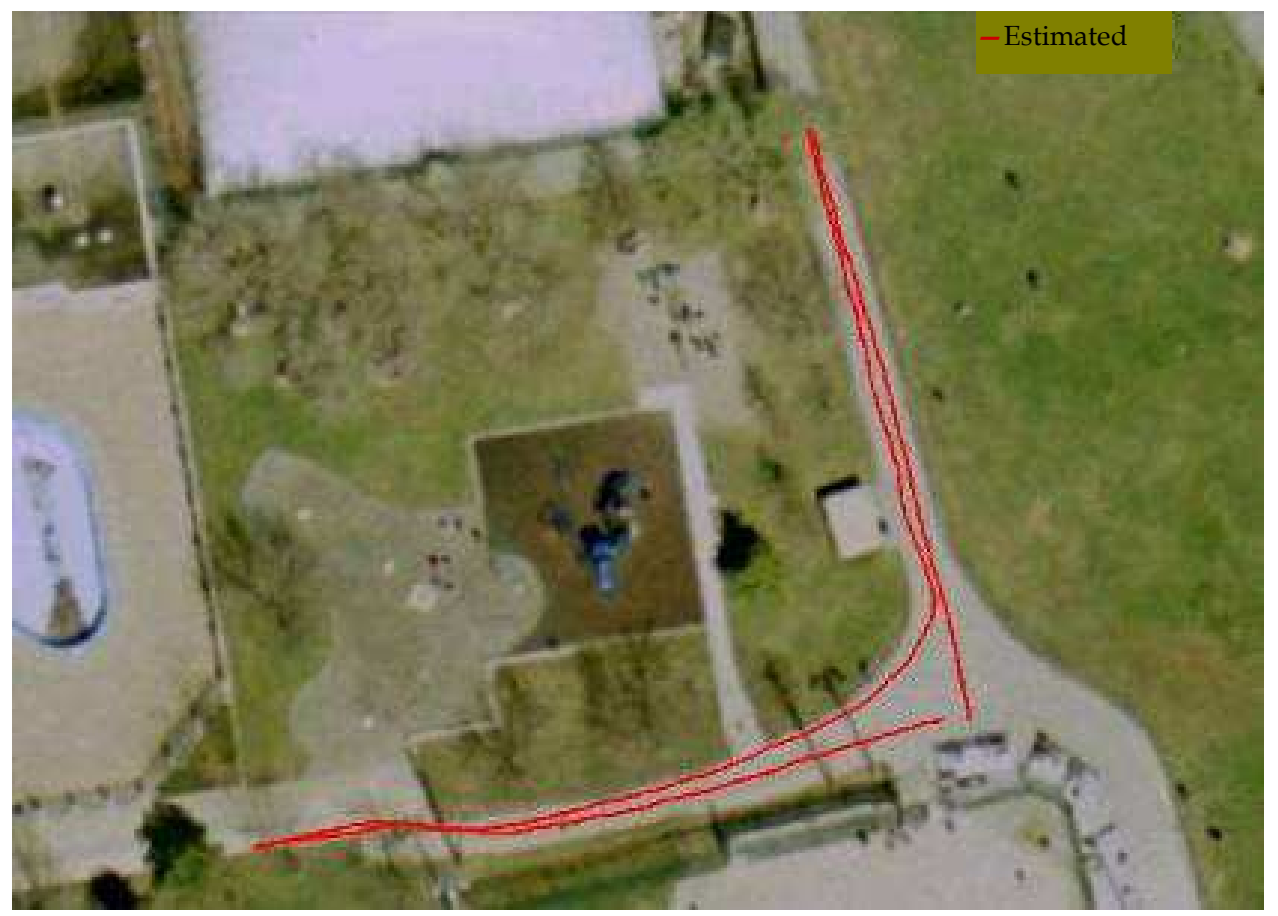

Fig. 8. Tracking the walk on a J-shaped path at a park

In the setup of experiments related to Fig 8, the walk is along an outdoor J-shaped path near a playground. The plotted trace recorded is similar to the actual path walked.

\section{Sensor fusion and Kalman filtering}

\subsection{Sensor fusion}

The use of higher quality inertial/magnetic sensor modules will improve positioning accuracy but the cost of such a solution is often much higher. An alternative solution is to use various positioning data from multiple sources such as radio navigation aids and additional sensors onboard. Through the control applied to the drift error, a positioning system using multiple data sources "fused" together is able to outperform a system using a single data source. Complementary filtering techniques were used to integrate data from different positioning aids in the past. Nowadays, Kalman filtering is popular in combination of two estimates of a variable. For example, IPS data can be integrated with GPS data to provide enforced positioning ability in both indoor and outdoor environment.

\subsection{Kalman filter}

Although dynamical behavior of a linear system is often expressed in the form of continuous differential equations, in practice, the measurements are provided at discrete intervals of time. The form of difference equations are used as below: 


$$
\mathbf{x}_{k}=\mathbf{F}_{k-1} \mathbf{x}_{k-1}+\mathbf{B}_{k-1} \mathbf{u}_{k-1}+\mathbf{w}_{k-1}
$$

where the $n$ vector, $\mathbf{x}_{k}$, is called the state of the system at time $k, \mathbf{u}_{k-1}$ is a $p$ vector of deterministic inputs and $\mathbf{w}_{k-1}$ is the system noise at time $k-1 . \mathbf{F}_{k-1}$ is an $n \times n$ state transition matrix from time $k-1$ to time $k$, known as the system matrix, and $\mathbf{B}_{k-1}$ is an $n \times p$ system input matrix. $\mathbf{F}_{k-1}$ and $\mathbf{B}_{k-1}$ are constant or time varying matrices. The system (or process) noise $\mathbf{w}_{k}$ is normally distributed with zero mean and a power spectral density of $\mathbf{Q}_{k-1}$.

The vector $\mathbf{x}_{k}$ contains all the information regarding the present state of the system, but cannot be measured directly. Instead, only the system's $m$ vector, $\mathbf{z}_{k}$, is available. This measurement vector is a linear combination of the state, $\mathbf{x}_{k}$, that is corrupted by the measurement noise $\mathbf{v}_{k}$. This can be expressed in terms of the system state by the following equation:

$$
\mathbf{z}_{k}=\mathbf{H}_{k} \mathbf{x}_{k}+\mathbf{v}_{k}
$$

where $\mathbf{H}_{k}$ is an $m \times n$ measurement matrix. The measurement (or observation) noise, $\mathbf{v}_{k}$, has zero-mean and is normally distributed, with power spectral density $\mathbf{R}_{k}$.

The Kalman filter for the system described here seeks to provide the best estimates of the states, $\mathbf{x}_{k}$, using the measurements, $\mathbf{z}_{k}$, model of the system provided by the matrices $\mathbf{F}_{k}, \mathbf{B}_{k}$ and $\mathbf{H}_{k}$, and knowledge of the system and measurement statistics given in the matrices $\mathbf{Q}_{k}$ and $\mathbf{R}_{k}$.

From (23) and (24), we can recognize that Kalman filter is a recursive filter: only the state of last time step and the current measurement are used. The estimation is conceptualized as two distinct phases, "Predict" and "Update", described by two distinct set of equations. In the first phase, the prediction is based on previous best estimate. The result is called a priori state estimate. In the second phase, the updating is done based on the a priori state estimate with new measurement. The result is a posteriori state estimate. The two set of distinct equations are as below:

\section{The prediction process}

Predicted (a priori) state estimate is the best estimate of the state at time $k-1$, denoted as $\mathbf{x}_{k-1 / k-}$ 1 . Since the process noise, $\mathbf{w}_{k-1}$, is normally distributed with zero-mean, the best prediction of is given by

$$
\mathbf{x}_{k / k-1}=\mathbf{F}_{k-1} \mathbf{x}_{k-1 / k-1}
$$

while the predicted (a priori) estimate covariance at time $k$ predicted at time $k-1$, is given by

$$
\mathbf{P}_{k / k-1}=\mathbf{F}_{k-1} \mathbf{P}_{k-1 / k-1} \mathbf{F}_{k-1}^{\mathrm{T}}+\mathbf{Q}_{k-1}
$$

\section{The measurement update}

On the arrival of new measurement $\mathbf{z}_{k}$, at time $k$, it is compared with the a priori state estimate of the measurement. The measurement is then used to update the prediction to generate a best estimate, i.e., the a posteriori state estimate at time $k-1$ :

$$
\mathbf{x}_{k / k}=\mathbf{x}_{k / k-1}-\mathbf{K}_{k}\left[\mathbf{H}_{k} \mathbf{x}_{k / k-1}-\mathbf{z}_{k}\right]
$$


Accordingly, the updated (a posteriori) estimate covariance is:

$$
\mathbf{P}_{k / k}=\mathbf{P}_{k / k-1}-\mathbf{K}_{k} \mathbf{H}_{k} \mathbf{P}_{k / k-1}
$$

where the optimal Kalman gain matrix is given by:

$$
\mathbf{K}_{k}=\mathbf{P}_{k / k-1} \mathbf{H}_{k}^{\mathrm{T}}\left[\mathbf{H}_{k} \mathbf{P}_{k / k-1} \mathbf{H}_{k}^{\mathrm{T}}+\mathbf{R}_{k}\right]
$$

Typically, the two phases alternate, with the prediction advancing the state until the next scheduled measurement, and the update incorporating the measurement.

\subsection{Extended Kalman filter}

Kalman filter is optimal in either the least square sense or maximum likelihood sense if it is applied to linear dynamic system with zero mean, normally distributed noise. Kalman filter becomes not optimal when the system is nonlinear, or the noise is not normally distributed.

EKF is the nonlinear version of the Kalman filter. EKF linearizes the dynamic system about the current mean and covariance. The estimate is suboptimal, i.e. it is an approximation of the optimal estimate.

In EKF, the state transition and measurement models may be non-linear differentiable functions:

$$
\begin{gathered}
\mathbf{x}_{k}=f\left(\mathbf{x}_{k-1}, \mathbf{u}_{k-1}\right)+\mathbf{w}_{k-1} \\
\mathbf{z}_{k}=h\left(\mathbf{x}_{k}\right)+\mathbf{v}_{k}
\end{gathered}
$$

where system noise $\mathbf{w}_{k}$ is normally distributed with zero mean and covariance $\mathbf{Q}_{k}$ while measurement noise $\mathbf{v}_{k}$ is zero mean normally distributed with covariance $\mathbf{R}_{k}$. The functions $f$ and $h$ can be used to estimate the current state and measurement from previous state and measurement respectively. However, the current covariance of system noise and measurement noise cannot be estimated from these two functions directly. Instead the Jacobians, matrices of partial derivatives, must be calculated. The state transition and measurement matrices are defined as:

$$
\begin{gathered}
\mathbf{F}_{k-1}=\left.\frac{\partial f}{\partial \mathbf{x}}\right|_{\mathbf{x}_{k-1 / k-1}, \mathbf{u}_{k-1}} \\
\mathbf{H}_{k}=\left.\frac{\partial h}{\partial \mathbf{x}}\right|_{\mathbf{x}_{k / k-1}}
\end{gathered}
$$

Similar with Kalman filter, each EKF iteration is also composed of two distinct phases, representing by two set of equations as below:

\section{The prediction process}

Predicted state estimate and its covariance through linearization of system dynamics and application of the prediction step of the Kalman filter 


$$
\begin{gathered}
\mathbf{x}_{k / k-1}=f\left(\mathbf{x}_{k-1 / k-1}, \mathbf{u}_{k-1}\right) \\
\mathbf{P}_{k / k-1}=\mathbf{F}_{k-1} \mathbf{P}_{k-1 / k-1} \mathbf{F}_{k-1}^{T}+\mathbf{Q}_{k-1}
\end{gathered}
$$

\section{The measurement update}

Measurement residual is calculated through linearization of system dynamics

$$
\mathbf{y}_{k}=\mathbf{z}_{k}-h\left(\mathbf{x}_{k / k-1}\right)
$$

The relevant covariance is

$$
\mathbf{S}_{k}=\mathbf{H}_{k} \mathbf{P}_{k / k-1} \mathbf{H}_{k}^{\mathrm{T}}+\mathbf{R}_{k}
$$

The suboptimal Kalman gain is

$$
\mathbf{K}_{k}=\mathbf{P}_{k / k-1} \mathbf{H}_{k}^{\mathrm{T}} \mathbf{S}_{k}^{-1}
$$

The state estimate is updated as

$$
\mathbf{x}_{k / k}=\mathbf{x}_{k / k-1}+\mathbf{K}_{k} \mathbf{y}_{k}
$$

The relevant estimate covariance is also updated as

$$
\mathbf{P}_{k / k}=\left(\mathbf{I}-\mathbf{K}_{k} \mathbf{H}_{k}\right) \mathbf{P}_{k / k-1}
$$

\subsection{Application examples}

Kalman filter, sometimes with other techniques, have already been used for data fusion and positioning. Recently, Zhao \& Wang (2011) use EKF for the fusion of data from inertial sensors, ultrasonic sensors, and magnetic sensor. A 3D magnetic sensor and a 3D accelerometer are combined to measure the gravity and the earth's magnetic field for the static orientation. A 3D gyroscope is used to obtain the dynamic orientation. The integral drift of accelerometer is periodically calibrated by an ultrasonic sensor. All these data are fused by EKF. The measurements are the position obtained by ultrasonic sensor and the orientation obtained by magnetic sensors and accelerometers. The states incorporated the position, velocity, and orientation. The experimental results demonstrated that the uncertainty of orientation and position is lowered.

In another research, Shen et al (2011) apply Kalman filter, together with Fast Orthogonal Search (FOS) in the 2D navigation for land vehicles. Kalman filter is used for data fusion of GPS and inertial sensors, i.e. the integration of GPS, IMU and vehicle built-in odometer. FOS is a nonlinear error identification technique. It is used to model non-stationary stochastic sensor errors and non-linear inertial errors in the study. The road test trajectories confirm that a module with both Kalman filtering and FOS technique outperforms that with Kalman filtering only.

It can be concluded that improvement of positioning accuracy will be possible if data from multiple sensors, including magnetic sensors, are fused by Kalman filter. Further improvement is achievable if Kalman filtering is combined with other techniques. 


\section{Summary}

In this chapter, we firstly defined local positioning systems and introduced their applications. Then we presented strap-down inertial positioning techniques covering not only the concepts of dead reckoning and inertial position, but also the algorithms, especially for the rotation of reference frames and the tracking of the orientation of a moving object. We also evaluated the performances of inertial sensors, i.e., gyroscopes and accelerometers and pointed out that the increasing integral drift over time is the main problem affecting the accuracy of positioning. As an example among the efforts to solve this problem, we proposed an approach to integrate pedestrian dead reckoning with inertial positioning in precise tracking, where the characteristics of human gaits are utilized. Magnetic sensors have to be incorporated to update the orientation of moving objects and the relevant algorithm is provided. The experimental study was also included to demonstrate the implementation and the result of positioning. Finally, among existing solutions to enhance the accuracy of local positioning, Kalman filter, EKF and their algorithms are introduced and the examples of their applications are also presented. Magnetic sensors are important resources of data to be fused through Kalman filtering.

\section{References}

Huang, C.; Liao, Z. \& Zhao, L. (2010). Synergism of SIN and PDR in Self-contained Pedestrian Tracking. IEEE Sensor Journal, Vol. 10, No. 8, pp1349-1359.

McBrewster, J.; Miller, F. P. \& Vandome,A. F. (2009). Navigation: Latitude, Longitude, Dead Reckoning, Pilotage, Celestial Navigation, Marine Chronometer, Sextant, Inertial Navigation System, Radio Navigation, Satellite System, Passage Planning. Alphascript Publishing.

Mezentsev, O.; Lachapelle, G. \& Collin, J. (2005). Pedestrian Dead Reckoning - A Solution to Navigation in GPS Signal Degraded Areas. Geomatica, Vol. 59, No.2, pp. 175-182.

Rose, J. \& Gamble, J. (1994). Human Walking, 2nd Ed. Williams \& Wilkins.

Sarton, G. \& George, A. (1959). History of Science: Hellenistic Science and Culture in the Last Three Centuries B.C. New York: The Norton Library, Norton \& Company Inc..

Shen, Z.; Georgy, J.; Korenberg, M. J. \& Noureldin, A. (2011). Low Cost Two Dimension Navigation Using an Augmented Kalman Filter/Fast Orthogonal Search Module for the Integration of Reduced Inertial Sensor System and Global Positioning System. Transportation Research. Part C, Emerging Technologies, vol. 19, no. 6, pp.1111-1132.

Stirling, R. \& Fyfe, K. (2005). Evaluation of a New Method of Heading Estimation for Pedestrian Dead Reckoning Using Shoe Mounted Sensors. The Journal of Navigation, vol.58, pp. 31-45, The Royal Institute of Navigation.

Thiessen, F. J. \& Dales, D. N. (1983). Automotive Steering, Suspension and Braking Systems, Reston Pub. Co.

Woodman, O. \& Harle, R. (2008). Pedestrian Localisation for Indoor Environments. Proceedings of the Tenth International Conference on Ubiquitous Computing (UbiComp 08), Seoul, Korea, ACM, Sep 2008.

Zhao, H. \& Wang, Z. (2011). Motion Measurement Using Inertial Sensors, Ultrasonic Sensors, and Magnetometers with Extended Kalman Filter for Data Fusion, IEEE Sensors Journal, vol. pp, no. 99, pp. 1-8. 


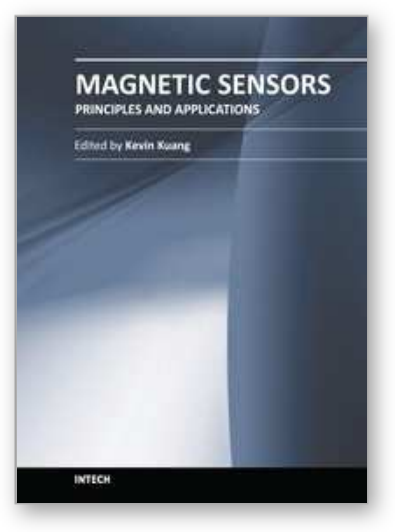

\author{
Magnetic Sensors - Principles and Applications \\ Edited by Dr Kevin Kuang
}

ISBN 978-953-51-0232-8

Hard cover, 160 pages

Publisher InTech

Published online 09, March, 2012

Published in print edition March, 2012

This book provides an introductory overview of the research done in recent years in the area of magnetic sensors. The topics presented in this book range from fundamental theories and properties of magnets and their sensing applications in areas such as biomedicine, microelectromechanical systems, nano-satellites and pedestrian tracking. Written for the readers who wished to obtain a basic understanding of the research area as well as to explore other potential areas of applications for magnetic sensors, this book presents exciting developments in the field in a highly readable manner.

\title{
How to reference
}

In order to correctly reference this scholarly work, feel free to copy and paste the following:

Chengliang Huang and Xiao-Ping Zhang (2012). The Application of Magnetic Sensors in Self-Contained Local Positioning, Magnetic Sensors - Principles and Applications, Dr Kevin Kuang (Ed.), ISBN: 978-953-51-0232-8, InTech, Available from: http://www.intechopen.com/books/magnetic-sensors-principles-and-applications/theapplication-of-magnetic-sensors-in-self-contained-local-positioning

\section{INTECH}

open science | open minds

\section{InTech Europe}

University Campus STeP Ri

Slavka Krautzeka 83/A

51000 Rijeka, Croatia

Phone: +385 (51) 770447

Fax: +385 (51) 686166

www.intechopen.com

\section{InTech China}

Unit 405, Office Block, Hotel Equatorial Shanghai

No.65, Yan An Road (West), Shanghai, 200040, China

中国上海市延安西路65号上海国际贵都大饭店办公楼405单元

Phone: +86-21-62489820

Fax: $+86-21-62489821$ 
(C) 2012 The Author(s). Licensee IntechOpen. This is an open access article distributed under the terms of the Creative Commons Attribution 3.0 License, which permits unrestricted use, distribution, and reproduction in any medium, provided the original work is properly cited. 